\title{
Desafíos socioeducativos de las comunidades de tratamiento de la juventud en conflicto con la ley. Motivación y buenas prácticas de instrucción y gestión institucional. Dos casos de México
}

\author{
Socio-Educational Challenges of Communities Treating Young People in Conflict with the \\ Law. Motivation and Good Practices of Instruction and Institutional Management. Two \\ Cases from Mexico
}

\section{Desafios socioeducativos das comunidades de tratamento da juventud em conflito com a lei. Motivação e boas práticas de instrução e gestão institucional. Dois casos de México}

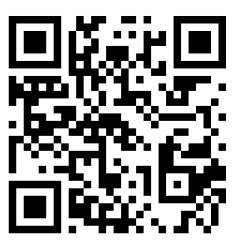

\author{
Carlos Acevedo-Rodríguez \\ Centro de Cooperación Regional para la Educación de Adultos en América Latina y el Caribe \\ Michoacán, México \\ carlos.acevedo@crefal.org \\ http://orcid.org/0000-0002-4011-9626
}

Recibido • Received • Recebido: 10 / 01 / 2019

Corregido • Revised • Revisado: 26 / 06 / 2020

Aceptado • Accepted • Aprovado: 18 / 08 / 2020

\begin{abstract}
Resumen: En México, la juventud adolescente que ha tenido un conflicto con la ley y ha sido juzgada y sentenciada a realizar medidas en libertad o en encierro $(J C L)$ debe asistir o vivir en espacios institucionales denominados comunidades. En estos espacios se imparten diversas actividades de rehabilitación, como terapias psicológicas, talleres artísticos y laborales, y clases escolares. En 2016 se promulgó la Ley Nacional del Sistema Integral de Justicia Penal para Adolescentes (LNSIJPA) (Cámara de Diputados de H. Congreso e la Unión, 2016). El objetivo es transitar de un modelo punitivo de atención a otro de carácter socioeducativo. Actualmente se carece de información sobre cómo las comunidades se adaptan a lo planteado en la Ley, específicamente respecto a las buenas prácticas de instrucción y gestión institucional. En el presente artículo científico se muestran los resultados del análisis cualitativo de dos comunidades ubicadas en la zona centro de México. A partir de entrevistas a treinta personas jóvenes sujetas a la LNSIJPA, entrevistas y grupos de discusión con veinte personas funcionarias, y observación de actividades socioeducativas; se identificó el perfil de la JCL y las buenas prácticas que se llevan a cabo. Se constató que, a pesar de diversos problemas y desventajas de la JCL, se realizan buenas prácticas que impactan positivamente en la motivación de jóvenes y personas funcionarias. Para ilustrar este proceso, se construye una perspectiva analítica de la influencia de la motivación en el aprendizaje y gestión. Finalmente, sostenemos que cada persona tiene la mejor capacidad para aprender en ambientes sociales que estimulan la motivación, y se presentan áreas de mejora.
\end{abstract}

Palabras claves: Educación correccional; eficacia del centro de enseñanza; gestión del centro de enseñanza; motivación para el aprendizaje; enseñanza y formación. 
http://doi.org/10.15359/ree.24-3.20

http://www.una.ac.cr/educare

educare@una.ac.cr

\begin{abstract}
In Mexico, adolescents who have had conflicts with the law and have been tried and sentenced to serve time in freedom or confinement must attend or live in institutions called communities. In these spaces, a variety of activities, such as psychological therapies, artistic and labor workshops, and classes are carried out as part of young people's rehabilitation. In 2016, the National Law of the Comprehensive Criminal Justice System for Adolescents was enacted (Cámara de Diputados de H. Congreso de la Unión, 2016). The objective is to move from a treatment based on a punitive model to a strategy based on socio-educational improvements. Currently, there is not enough information about how the communities are adapting to the new guidelines, specifically regarding best practices of instruction and institutional management. This scientific paper shows the results of the qualitative analysis of two communities located in the central zone of Mexico. Through interviews with thirty young people serving their sentences under the new law, interviews and discussion groups with twenty institutional officials, and observation of socio-educational activities, the profiles of youths in these communities and best practices were identified. It was found that despite several problems and disadvantages of young people, the best practices identified have a positive impact on the motivation of young people and officials. To illustrate this process, an analytical framework about the influence of motivation on learning and management was constructed. Finally, we state that every person has the best capacity to learn in social environments which are able to stimulate motivation; we also propose areas of improvement.
\end{abstract}

Keywords: Correctional education; effectiveness of the teaching center; management of the teaching center; learning motivation; education and training.

Resumo: No México, o adolescente jovem que teve algum conflito com a lei, foi julgado e sentenciado a executar medidas em liberdade ou na prisão (JLC) deve frequentar ou viver em espaços institucionais denominados comunidades. Nesses espaços, há diversas atividades de reabilitação, como terapias psicológicas, oficinas artísticas e técnicas, e classes escolares. Em 2016, foi promulgada a Lei Nacional do Sistema Integral de Justiça Penal para Adolescentes (LNSIJPA) (Cámara de Deputados de H. Congresso e da União, 2016). O objetivo é transitar um modelo punitivo de atenção a um outro com finalidade socioeducativa. Atualmente, faz falta informações sobre como as comunidades se adaptam ao que diz a Lei, especificamente relacionadas às boas práticas de instrução e gestão institucional. No presente artigo científico, são mostrados os resultados da análises qualitativa de duas comunidades localizadas na zona central do México. A partir de entrevistas e trinta jovens sujeitos à LNSIJPA, entrevistas e grupos de discussão com vinte pessoas funcionarias, e observação de atividades socioeducativas; foi identificado o perfil da $\mathrm{JCL}$ e das boas práticas realizadas. Foi constatado que, apesar dos diversos problemas e desvantagens da JCL, forma realizadas boas praticas que tiveram um impacto positivo na motivação dos jovens e das pessoas funcionarias. Para ilustrar esse processo, foi construída uma perspectiva analítica sobre a influência da motivação na aprendizagem e na gestão. Por fim, mantemos que cada pessoa tem melhor capacidade de aprender em ambientes sociais que estimulam a motivação, e são apresentadas áreas de melhoria.

Palavras-chave: Educação prisional; eficiência do centro de ensino; gestão do centro de ensino; motivação para aprender; educação e formação. 


\section{Introducción}

En América Latina, Estados Unidos y Europa, la juventud adolescente que ha tenido algún tipo de conflicto con la ley, que ha sido juzgada y sentenciada a llevar a cabo sus medidas, ya sea en libertad o en encierro (JCL), debe asistir o vivir en espacios institucionales con características de reformatorios o correccionales. En estos espacios se llevan a cabo una serie de actividades que se contemplan como parte de la rehabilitación, entre ellas terapias psicológicas, talleres artísticos y laborales, y clases escolares de nivelación y finalización de grados educativos.

En México, los reformatorios que atienden a la JCL se conocen con el nombre de comunidades de tratamiento de adolescentes en conflicto con la ley. De acuerdo con la Ley Nacional del Sistema Integral de Justicia Penal para Adolescentes (LNSIJPA) (Cámara de Diutados de H. Congreso e la Unión, 2016), la población que atiende el Sistema Integral de Justicia Penal para Adolescentes es definida como aquella que al momento de cometer un delito se encuentra entre los doce y diecisiete años de edad. Por ninguna circunstancia, la persona adolescentes de doce y trece años puede ser sancionada penalmente (quedan al cuidado de quien legalmente corresponda). La opción de encarcelamiento se reserva para los delitos más graves (homicidio, secuestro, violación, narcotráfico, terrorismo, robo con violencia). El tiempo máximo de dictado de sentencia, luego de la detención, es de seis meses (Art. 117), y el tiempo máximo de detención es de cinco años (Art. 145). Las personas adolescentes que cumplan la mayoría de edad en el transcurso de sus medidas siguen siendo parte del Sistema Integral de Justicia Penal para Adolescentes, debido a que cometieron los delitos cuando eran adolescentes (Art. 1).

En estas comunidades se ofrece una serie de tratamientos y terapias, entre estos se hallan sesiones con psicólogas, terapeutas de aprendizaje, asistentes sociales, talleres de capacitación laboral y promoción de creatividad, y se contempla la asistencia a clases formales de educación en espacios que se denominan escuelas. En la escuela se desempeñan pedagogas que buscan nivelar al estudiantado y prepararlo para realizar exámenes que acrediten la finalización de un grado escolar.

No obstante estos apoyos, las comunidades se han caracterizado por trabajar con un enfoque punitivo que no responde al respeto de los derechos de niñas, niños y adolescentes (González Ibarra y Reyes Barragán, 2007). Precisamente, en el año 2016 se promulga la LNSIJPA, entre sus principales objetivos se halla transitar de un modelo de atención a la JCL que subraya aspectos punitivos a otro que se enmarque en un modelo socioeducativo con énfasis en la reintegración social y familiar. Así, las comunidades de atención deben adaptarse a lo que establece la LNSIJPA.

Debido a que las comunidades son bastante cerradas, se ha dificultado el análisis científico de lo que ocurre al interior de estas. Por ende, se carece de información sobre cómo las comunidades se adaptan a lo planteado en la LNSIJPA, y respecto a las buenas prácticas de instrucción y gestión institucional contenidas en las actividades socioeducativas que se llevan 
http://doi.org/10.15359/ree.24-3.20

http://www.una.ac.cr/educare

educare@una.ac.cr

a cabo con la JCL. Con buenas prácticas se entienden actividades que promueven resultados socioeducativos que no se limitan a pruebas estandarizadas sino que se expresan en avances en aspectos emocionales y actitudinales. Es decir, prácticas que repercuten positivamente en el desempeño de jóvenes y funcionariado, base del logro y aprovechamiento socioeducativo.

Contar con información de buenas prácticas sería útil para registrar los avances en los objetivos de la LNSIJPA, así como para que autoridades, funcionarias y funcionarios de las comunidades pueden adaptar las buenas prácticas a sus contextos particulares. Por ende, otorgar insumos para propiciar en mayor medida un ambiente socioeducativo y de integración en las comunidades.

El presente artículo busca aportar en enfrentar estos vacíos a partir del análisis de dos comunidades, de las cuales no se especificará nombre ni lugar para mantener la confidencialidad a la que el autor se comprometió cuando negoció el acceso. Solo se señalará que ambas comunidades se encuentran en la misma ciudad ubicada en la zona centro de México.

Antes de concretar el trabajo de campo, se revisó la bibliografía nacional e internacional especializada en la temática de atención institucional a la JCL. Con esa base, el trabajo de campo tuvo como referentes el perfil educativo del estudiantado, y las dimensiones de instrucción y gestión institucional. A medida que se avanzó en la investigación, se consideró pertinente recurrir a la perspectiva analítica de motivación, tanto para el aprendizaje de la JCL como para el trabajo de las personas funcionarias.

En la investigación desarrollada se constató que, a pesar de diversos problemas y desventajas de la JCL, sí se llevan a cabo una diversidad de buenas prácticas. En este sentido, la presente investigación se considera un aporte en la línea de que es posible generar condiciones sociales para lograr el aprovechamiento socioeducativo en cualquier estudiantado.

A continuación, se ahonda en la problemática de investigación, objetivos y metodología;se desarrolla una síntesis de los principales planteamientos de bibliografía nacional e internacional, y se presentan los hallazgos de la investigación de campo. En este último punto, se profundiza el contexto de las comunidades y el perfil de la JCL atendida, para luego ahondar en las buenas prácticas instruccionales y de gestión identificadas en diversas actividades.

Acto seguido, y para comprender el proceso subyacente a las buenas prácticas, se desarrolla una perspectiva analítica de la influencia de la motivación en los procesos de aprendizaje y gestión.

Finalmente, se presentan las conclusiones y áreas de mejora.

\section{Problemática, objetivos y metodología}

La reforma de 2006 del Artículo 18 Constitucional adoptó principios e instrumentos internacionales para crear sistemas integrales de justicia para jóvenes adolescentes. Esta reforma 
http://doi.org/10.15359/ree.24-3.20

adquiere cuerpo completo en 2016, en la Ley Nacional del Sistema Integral de Justicia Penal para Adolescentes (LNSIJPA) (Cámara de Diutados de H. Congreso e la Unión, 2016). Retomando los derechos de niñas, niños y adolescentes, en la LNSIJPA se establece una normativa que apunta a transitar de un sistema punitivo a otro socioeducativo.

Con este espíritu, en la LNSIJPA se plantea que la atención a JCL tiene como objetivo la reintegración social y familiar y se establece en el Art. 28 que "la reintegración se llevará a través de diversos programas socioeducativos de intervención destinados a incidir ... en los ámbitos familiar, escolar, laboral y comunitario" (Cámara de Diutados de H. Congreso de la Unión, 2016, p. 8).

Uno de los principales instrumentos para dar cuenta de la exigencia socioeducativa es el Plan Individualizado de Ejecución (PIE). EI PIE (Cámara de Diutados de H. Congreso e la Unión, 2016) especifica:"Los objetivos particulares que se pretenden cumplir durante el proceso ... [así como] la determinación de las actividades educativas, deportivas, culturales, laborales o formativas en las que participará" (p. 57). El Plan es diseñado por el personal jurídico y técnico (personal de pedagogía, terapias, psicología, trabajo social) junto con la persona joven adolescente y sus familiares, y es aprobado por un juez de ejecución.

La educación adquiere tal relevancia que en el Art. 51 de la LNSIJPA (Cámara de Diutados de H. Congreso de la Unión, 2016) se reconoce el derecho de las personas adolescentes "a cursar el nivel educativo que le corresponda y a recibir instrucción técnica o formación práctica sobre un oficio, arte o profesión y enseñanza e instrucción en diversas áreas del conocimiento" (p. 14).

Sin embargo, tales planteamientos socioeducativos se proponen en un contexto de atención a la JCL que se ha caracterizado por un enfoque punitivo que no responde al respeto de los derechos de niñas, niños y adolescentes (González lbarra y Reyes Barragán, 2007). Asimismo, es conocido que estos grupos de jóvenes reciben educación escolar con modelos educativos que no se consideran sólidos ni de buena calidad (Azaola, 2015), y solo dedican de dos o tres veces a la semana a tal actividad (Centro de Investigaciones y Estudios Superiores en Antropología Social [CIESAS], 2016). Por otra parte, se desconocen los procesos en que se inscribe la educación en otras áreas, como la artística y laboral, así como la gestión de las actividades socioeducativas.

Debido a que las comunidades son bastante cerradas, se ha dificultado el análisis científico de lo que ocurre al interior de estas. En consecuencia, se carece de información sobre cómo las comunidades se adaptan al carácter socioeducativo contenido en la LNSIJPA, y respecto a qué prácticas de instrucción y gestión institucional estarían logrando resultados positivos con la JCL.

Para responder a estas problemáticas, se realizó un análisis de dos comunidades, de las cuales no se especificará nombre ni lugar para mantener la confidencialidad a la que el autor se comprometió a la hora de obtener acceso. Al respecto, solo se señalará que ambas comunidades se encuentran en la misma ciudad ubicada en la zona centro de México. 
http://doi.org/10.15359/ree.24-3.20

http://www.una.ac.cr/educare

educare@una.ac.cr

En una comunidad, jóvenes hombres cumplen sentencias en encierro (internamiento) mientras en la otra, jóvenes mujeres y hombres que se encuentran en libertad, deben asistir cierta cantidad de veces a la semana a la comunidad para llevar a cabo una serie de actividades (externación). En estas comunidades el autor del artículo más otros dos investigadores, realizaron:

- Entrevista individual formal semiestructurada a todas las personas funcionarias de cada comunidad y tres grupos de discusión con el área educativa de cada institución. En cada comunidad laboran diez personas funcionarias (directora o director, pedagogas, terapeutas de aprendizaje, talleristas, psicólogas, guardias).

- Entrevista individual formal semiestructurada a la población total de la comunidad de internamiento, veinte jóvenes hombres. Todos cumplen sus medidas en el marco de la LNSIJPA.

- Entrevista individual formal semiestructurada ajóvenes dela comunidad deexternación, siete hombres y tres mujeres. Todas estas personas jóvenes cumplen sus medidas en el marco de la LNSIJPA.

- Observación de las diversas actividades socioeducativas que tienen lugar en estos recintos: talleres artísticos y laborales, terapias psicológicas, terapias de aprendizaje, clases escolares.

- Lectura de "Plan individualizado de ejecución (PIE)" de algunas personas jóvenes entrevistadas. En cada institución se tuvo acceso a los PIE por un tiempo aproximado de una hora (debido a medidas de seguridad, pues los PIE se archivan junto a información delicada que incluye expedientes de delitos, entre otros).

- En la observación de actividades socioeducativas se realizó una serie de entrevistas informales no estructuradas con la mayor cantidad de actores, JCL y personas funcionarias.

Debido a la restricción de ingresar a las comunidades cualquier aparato tecnológico de grabación de audio/video, el equipo investigador tomó notas en sus respectivos cuadernos de campo de: entrevistas, grupos de discusión y observación de actividades. Las visitas a las comunidades fueron de tres meses con una o dos visitas por semana. Esta temporalidad dio margen para que el equipo de investigación contrastara cada semana las notas tomadas, las categorías de análisis desarrolladas y las conclusiones alcanzadas.

A continuación, se presenta la revisión documental realizada previamente al trabajo de campo.

\section{Bibliografía internacional}

Además de provenir de entornos altamente desfavorecidos en términos socioeconómicos y muchas veces violentos, en las investigaciones internacionales se ha establecido que la población JCL cuenta con capacidades cognoscitivas que van de bajas a promedio, tiene retrasos importantes en lectura, matemática y lenguaje, y fracaso escolar (Foley, 2001). 
De acuerdo con las experiencias estudiadas en EE.UU., aproximadamente uno de cada tres jóvenes de estos grupos tiene un problema de aprendizaje identificado (Magee Quinn et al., 2005) y otras estimaciones bordean el rango de $40 \%$ a $70 \%$ (Bullis et al., 2002). Asimismo, antes de ingresar al sistema judicial, gran parte de jóvenes acumula experiencias escolares negativas como absentismo, suspensión, deserción, expulsión, y otras formas de fracaso académico (Mathur y Schoenfeld, 2010; Wang et al., 2005). En esta línea, la gran mayoría de jóvenes ha abandonado la escuela uno o dos años antes de ingresar (Mathur y Schoenfeld, 2010; Wang et al., 2005).

La evidencia internacional muestra que los sistemas educativos que atienden a esta población son sistemas especiales $y$, sin embargo, estos sistemas se caracterizan por falta de pertinencia curricular y falta de motivación por parte de la juventud para tomar los cursos (Houchins et al., 2009).

También hay que agregar el problema con la autoridad que gran parte de jóvenes manifiesta (Nelson, 2000). Las investigaciones destacan que suelen tener problemas con la autoridad que se manifiesta de diversas formas: pasividad, indiferencia y, en ocasiones, desprecio (Nelson, 2000).

En cuanto a aspectos de gestión institucional, si bien escasea las investigaciones, se han desarrollado diversos estudios respecto a cómo afecta esta dimensión en la educación de jóvenes con problemas de aprendizaje y desórdenes de comportamiento y emocionales. En esta línea de trabajo, se ha constatado la importancia del liderazgo directivo y distribuido para propiciar una visión común y objetivos compartidos en torno al fin último de lograr la inclusión de esta población; y se ha destacado la importancia de profesionalizar constantemente al funcionariado que trabaja con jóvenes (Maccini y Calvin Cagnon, 2006).

Por otra parte, se ha mostrado la relevancia de desarrollar programas dirigidos a la población $\mathrm{JCL}$, altamente flexibles en términos de actividades y horarios (Atkins et al., 2005). Asimismo, se ha identificado la pertinencia de otorgar certificado laboral que acredite capacitaciones laborales, es decir, aumentar la probabilidad de una carrera en un trabajo que pague un salario decente y que tenga adecuada transferibilidad (Platt et al., 1996).

\section{Bibliografía nacional}

Los antecedentes que se presentan son recopilados de investigaciones que han aplicado principalmente encuestas a jóvenes que cumplen sus medidas en instituciones de internamiento o externación. Las investigaciones son las siguientes.

- En el año 2014 se realizó un estudio por Elena Azaola, en comunidades de internamiento de cuatro Estados de la República: Morelos, Hidalgo, Coahuila y Sinaloa. En esta investigación se aplicó una encuesta a 278 adolescentes con privación de libertad, 247 hombres y 31 mujeres (Azaola, 2015). 
http://doi.org/10.15359/ree.24-3.20

http://www.una.ac.cr/educare

educare@una.ac.cr

- Entre 2014 y 2016, el CIESAS Ilevó a cabo una investigación con juventud privada de su libertad en centros de internamiento de 17 entidades del país: Puebla, Morelos, Ciudad de México, Jalisco, Yucatán, Veracruz, Hidalgo, Zacatecas, Oaxaca, Durango, Coahuila, Sonora, Estado de México, Sinaloa, Chihuahua, Tabasco, Baja California. Se aplicó una encuesta a 730 jóvenes, 631 hombres y 99 mujeres (CIESAS, 2016).

- En 2017 se aplicó la Encuesta Nacional de Adolescentes en el Sistema de Justicia Penal (ENASJUP) realizada por el Instituto Nacional de Estadística y Geografía (INEGI) a 3308 jóvenes, hombres y mujeres, en las 53 comunidades de internamiento y externación de las 32 entidades federativas del país (INEGI, 2018). ${ }^{1}$

- En el año 2018, la Fundación Reinserta un Mexicano A.C., realizó una investigación en diez entidades federativas: Estado de México, Ciudad de México, Yucatán, Michoacán, Puebla, Tabasco, Guerrero, Veracruz, Nuevo León y Sinaloa. Se aplicó una encuesta a 502 adolescentes, 458 hombres y 44 mujeres, que en su momento se encontraban en internamiento (REINSERTA, 2018).

De acuerdo con estos estudios:

Para 2017, el $76.6 \%$ de la JCL se encuentra en externación y el $23.4 \%$ en internamiento (INEGI, 2018). Las edades de la JCL se concentran en los rangos de 16 a 17 años (25.5\%) y de 18 a 22 años (59.4 \%) (INEGl, 2018). El $75.9 \%$ de la población JCL tiene estudios de nivel básico al momento de la detención, el 22.1 \% educación media superior, y el 1.1 \% estudios universitarios (INEGl, 2018).

En 2016, del total de jóvenes en internamiento y externación, un 93 \% es hombre y un 7 $\%$ mujer. En la población internada, los hombres ascienden al $96 \%$ y las mujeres decaen al $4 \%$ (CIESAS, 2016).

Según los datos más recientes, el $21.6 \%$ de los hombres y el $20.5 \%$ de mujeres tienen al menos una hija o hijo ( $16.7 \%$ tiene una hija o hijo, $3.7 \%$ dos hijas o hijos, $1 \%$ más de tres hijas o hijos) (REINSERTA, 2018). En términos socioecocómicos, corresponde a una población de condiciones desfavorecidas. Al respecto, el $83.3 \%$ declaró provenir de familias que perciben un ingreso menor o igual a doce mil pesos mexicanos mensuales y un $61.4 \%$ declaró provenir de familias que perciben un ingreso menor o igual a siete mil pesos mexicanos (600 dólares y 350 dólares americanos respectivamente, para el tipo de cambio de 2018) (REINSERTA, 2018). ${ }^{2}$

\footnotetext{
${ }^{1}$ No se otorgan números respecto a la cantidad de hombres y mujeres encuestados.

2 De acuerdo con la Encuesta Nacional de Ingresos y Gastos de los Hogares (ENIGH) 2018, el ingreso promedio mensual por familia en México fue de 16536 pesos (INEGl, 2019). Unos 827 dólares americanos para el tipo de cambio de 2018.
} 
En la investigación elaborada por el CIESAS, se destacó que además de las desventajas socioeconómicas, los grupos de JCL están constituidos por personas cuyo proceso de socialización se desarrolla en un ambiente violento, tanto a nivel del barrio como familiar. En este sentido, se identificó que un 39 \% de esta población sufrió maltrato y no encontró ninguna ayuda (CIESAS, 2016). Asimismo, se constató una socialización en donde el consumo de drogas y alcohol es cotidiano y es común contar con familiares en prisión. Al respecto, más de la mitad de la JCL consumía drogas diariamente antes de ingresar a las comunidades (68 \%) o vivió en ambientes familiares de drogadicción o alcoholismo (30 \% y 57 \%), y tiene familiares en prisión (60\%) (CIESAS, 2016).

En la dimensión escolar, según el mismo estudio, se apreció que a la gran mayoría (72%) de JCL no le gustaba la escuela, pues la consideraban aburrida (CIESAS, 2016). En cuanto a la ENASJUP, un $39.3 \%$ de la JCL abandonó la escuela al menos una vez en su vida. En promedio, lo hicieron a la edad de 14 años (INEGI, 2018). Tal como se constató en el año 2018, entre las principales causas por las que dejan de estudiar, se tiene en orden de importancia: $30.5 \%$ por detención, $27.5 \%$ por desinterés, $27.3 \%$ para empezar a trabajar y $22.5 \%$ porque no le gustaba (REINSERTA, 2018). La gran cantidad de jóvenes estaba trabajando previamente a su incorporación al Sistema Integral de Justicia Penal para Adolescentes, $79.3 \%$ de los hombres y $75 \%$ de las mujeres (REINSERTA, 2018); lo cual puede ser una variable importante para dejar los estudios o tener complicaciones importantes para rendir en estos.

Si bien muchas de estas personas jóvenes cuentan con rezagos educativos, no se hallan cifras al respecto. No obstante, en el estudio de Azaola (2015) se constató que "no existen programas educativos sólidos, constantes y de buena calidad que permitan a los adolescentes continuar sus estudios mientras se encuentran internos" (p. 31). En la misma línea, en la investigación del CIESAS se observó que una vez que se recluyen en las comunidades, el tiempo que la población JCL dedica a la educación es de un par de horas dos o tres veces a la semana, considerado como insuficiente para nivelación y finalización de grados escolares (CIESAS, 2016).

No se tiene información respecto al funcionamiento de las restantes actividades socioeducativas, como talleres artísticos y laborales, y terapias psicológicas.

En general, en las investigaciones mencionadas se llama la atención al desinterés hacia el estudio, las condiciones económicas adversas de origen, condiciones de violencia y el bajo nivel escolar de los padres y madres, problemáticas que no promueven altas expectativas socioeducativas en la población JCL y dificultan la reintegración social.

Ahora bien, la gestión institucional consiste en las tareas de dirección que lleva a cabo la directora, director o equipo directivo de una institución. Al respecto, no se encontró información sistematizada sobre la gestión institucional que se lleva a cabo en las comunidades. No obstante, se puede señalar que en 2007 la formación del personal encargado de impartir los contenidos 
http://doi.org/10.15359/ree.24-3.20

http://www.una.ac.cr/educare

educare@una.ac.cr

socioeducativos, en su mayoría, no correspondía a una formación especializada para atender a la población JCL (González Ibarra y Reyes Barragán, 2007). No se tiene información sobre cómo ha evolucionado este fenómeno de formación.

Por otra parte, en diversos estudios de gestión institucional escolar a nivel nacional, se subraya la importancia de generar actividades orientadas a definir proyectos educativos consensuados, así como promover el trabajo colaborativo y el compromiso docente y el de madres y padres de familia en el proceso de enseñanza/aprendizaje que se lleva a cabo en la institución escolar (Acevedo et al., 2017). El efecto positivo en términos socioeducativos de estas actividades ocurre cuando logran un impacto en el espacio concreto en que tiene lugar el proceso de enseñanza/aprendizaje (Acevedo et al., 2017; Elmore, 2010; Fullan, 2010). Tales aspectos son promovidos por un liderazgo directivo y distribuido.

Con base en la bibliografía nacional e internacional revisada, consideramos que para las comunidades aplican los mismos lineamientos que se han constatado en las restantes organizaciones educativas.

\section{Hallazgos}

\section{Contexto adverso}

Al momento se realizar la investigación, la comunidad de internamiento atendía a una población total de veinte jóvenes hombres, todos sujetos a la LNSIJPA, mientras que la de externación atendía a diez personas en el marco de la LNSIJPA, siete hombres y tres mujeres. La población total de atención de la segunda institución, no sujeta a la LNSIJPA, era bastante mayor.

A partir de la reforma constitucional y de la promulgación de la LNSIJPA, la cantidad de población joven que cumple medidas descendió de forma pronunciada, de tal forma que en 10 años hubo una reducción de 90 \% de la población tratada en la comunidad de externación y de $50 \%$ en la comunidad de internamiento. En línea con los datos nacionales, aproximadamente el $90 \%$ de la población JCL tiene una edad que va de los 16 a 22 años y un $20 \%$ ha tenido al menos una hija o hijo. En la comunidad de externación un $12 \%$ son mujeres y la comunidad de internamiento solo atiende a jóvenes hombres. No se otorgará más información al respecto para mantener el anonimato de las instituciones analizadas.

El contexto donde se lleva a cabo el proceso de rehabilitación de los JCL de ambas comunidades analizadas conjuga una serie de elementos adversos. Si bien se identificaron diferencias entre comunidades, las problemáticas son, en gran parte, compartidas entre estas. A continuación se detallan tales problemáticas. Cuando sea relevante mencionar diferencias entre comunidades esto se hará de forma explícita. Por ende, si no se mencionan tales diferencias, esto quiere decir que los fenómenos son transversales a ambas instituciones. 
Respecto al ámbito educativo, en sintonía con la bibliografía, la gran mayoría de jóvenes que se entrevistaron ha abandonado la escuela uno o dos años antes de ingresar. Gran cantidad se dedicaba a actos delictivos de los cuales conseguían sumas de dinero considerables que no obtendrían en los trabajos a los que tiene acceso por sus niveles educativos y redes. Los actos delictivos son bastante variados y van desde robos hasta violaciones y homicidios. Se concentran los delitos más violentos en la comunidad de internamiento.

Algunas de estas personas jóvenes se veían ante la necesidad de robar para mantener sus hogares, pues se les cargaba con esa responsabilidad. Otras, si bien es común que realicen algún tipo de aporte económico a sus familias, no cargaban con dicha responsabilidad. Todo esto implica impactos negativos en la asistencia de las jóvenes y los jóvenes a la escuela y en el interés en ella. En este sentido, es común que jóvenes de las comunidades señalen que su experiencia escolar anterior les aburría, no les parecía importante o no representaba un reto. En muchos casos no asistían, pues se dedicaban a obtener dinero por vías ilegales o a pasar el tiempo con sus amistades.

Aproximadamente un $10 \%$ estaba cursando primaria, un $36 \%$ secundaria y un $54 \%$ preparatoria. Si bien estos niveles están bien identificados, debido a los rezagos educativos de esta población, en la observación de las clases escolares, se apreció que una clase del mismo grado se transforma en una de multigrado. Por ejemplo, en clase de secundaria, la pedagoga debe recurrir constantemente a contenidos de primaria. En línea con este aspecto y en sintonía con las investigaciones internacionales, en la observación de clases se apreció que la juventud tiene rezagos escolares básicos en lecto-escritura y en operaciones matemáticas. Esto fue confirmado por las pedagogas.

En aras de lograr una mejor identificación del perfil de aprendizaje de la JCL y de complicaciones que puedan tener en el proceso de aprendizaje, las terapeutas de aprendizaje señalaron que realizan los siguientes instrumentos de evaluación:

- BETA III. Mide aspectos de inteligencia no verbal como procesamiento de información visual y razonamiento espacial, velocidad de estos procesos, etc.

- NEUROPSI. Mide procesos cognoscitivos como nivel de conciencia, atención y concentración, memoria; lenguaje, habilidades visoespaciales, funciones ejecutivas, lectura, escritura, cálculo, etc.

- SHIPLEY 2. Se enfoca en la medición de capacidad intelectual en términos de adaptación a situaciones nuevas, abstracción, razonamiento lógico, establecimiento de relaciones o diferencias; compresión y uso de vocabulario, establecimiento de juicios y conclusiones, orientación espacial, etc. 
http://doi.org/10.15359/ree.24-3.20

http://www.una.ac.cr/educare

educare@una.ac.cr

En esta valoración se establece si hay algún problema de aprendizaje y si la persona joven es candidata a terapia individual o de taller. La terapia de taller se refiere a una terapia grupal, donde se reúnen grupos de jóvenes que tienen similitud en problemas de aprendizaje. Las terapeutas de aprendizaje comentaron que, a partir de estas evaluaciones, se ha detectado que una cantidad importante de la JCL tiene un problema de aprendizaje identificado. En este ámbito, señalaron que la población internada es la que más problemas de aprendizaje tiene, de modo que la gran mayoría de esta población (90\%) recibe terapias especiales de aprendizaje. En la población en externación un porcentaje menor recibe este tipo de tratamientos (20\%).

Las psicólogas y terapeutas de aprendizaje hacen ver que esta situación se debe a que las jóvenes y los jóvenes internados tienen mayores problemas de adicción a las drogas, donde las más comunes son el alcohol, la marihuana, los inhalantes (el activo), y la pasta base de cocaína. Esta problemática afecta más a los hombres debido al mayor consumo de drogas que registran previamente a su ingreso a las comunidades. Este dato fue coherente con lo comunicado por la $J C L$ en entrevistas formales e informales en donde la mayoría de los jóvenes en internamiento declaró consumir drogas previamente a su incorporación al Sistema Integral de Justicia Penal para Adolescentes.

Estas funcionarias también comentaron la situación de que en el período de gestación las madres tuvieron algún tipo de consumo de drogas o mala alimentación. En sintonía con los estudios, en general la población JCL proviene de familias muy desaventajadas en términos socioeconómicos y respecto a los niveles educativos que poseen sus familias.

Por otra parte, y en línea con la evidencia internacional y como un fenómeno que no se desarolla en la investigación nacional, se constató que no existe un modelo educativo diseñado para la población JCL. En estas comunidades, la JCL desarrolla sus clases escolares con otros modelos diseñados para otras poblaciones. Resaltan los modelos de educación para jóvenes y personas adultas del INEA, CEDEX y Preparatoria Abierta, entre otros modelos diseñados para poblaciones sin conflicto con la ley. De acuerdo con la observación de actividades escolares así como con las entrevistas con pedagogas, se apreció que el trabajo con estos modelos muestra diversas dificultades, entre ellas el no motivar a la JCL y no conseguir los resultados esperados. Esto último quedó en evidencia cuando las pedagogas plantearon que aproximadamente un $80 \%$ de la JCL reprueba los exámenes para los que está siendo preparada.

La falta de motivación es muy común en el ámbito de la escuela. En este espacio, observamos que prácticamente todo el estudiantado tiene importantes dificultades en prestar atención en clases, no realiza sus tareas y olvida muy fácilmente las materias enseñadas días anteriores. Así, la pedagoga pierde bastante tiempo en retomar contenidos anteriores que se suponen ya revisados. 
Las clases son bastante similares a las que recibe una persona joven que asiste a una escuela tradicional, es decir, tienen bastante dictado de definiciones y ejercicios memorísticos donde la pedagoga desempeña el rol central. Al conversar con estos grupos jóvenes, nos comunicaron que perciben la escuela como más de lo mismo, pues no difiere de la experiencia previa que poseen. El solo concepto de escuela adquiere forma en una representación negativa que no motiva y que causa rechazo en la JCL.

En cambio, en la observación de las actividades de terapias socioemocionales, de aprendizaje, y en los talleres artísticos y laborales muestran motivación. En esta línea, la JCL se expresa con bastante más entusiasmo cuando conversamos sobre sus actividades en las terapias y talleres.

No obstante, si bien se otorgan una serie de talleres de capacitación, estos talleres laborales no requieren elevada formación, no se otorgan certificados profesionales y no se cuenta con sistemas de continuidad. Estos aspectos fueron destacados por la JCL y personas funcionarias.

En relación con las terapias psicológicas, en la observación del trabajo de psicólogas con jóvenes, se apreció una cantidad considerable de jóvenes que sufre problemas de autoestima y problemas de atención/concentración, y se constató un serio déficit general en cuanto a la capacidad de proyectarse en el tiempo, de generar planes de vida que contengan metas y plantarse medios para conseguirlos. Las psicólogas confirman que este fenómeno es general en la población atendida y que es común que la JCL viva el día a día o que tenga metas muy cercanas en el tiempo, como conseguir un certificado de estudios de primaria, secundaria o preparatoria y, en el ámbito legal, trabajar en empleos que no requieren altos niveles de educación o capacitación laboral.

Sobre los elevados problemas con respetar a la autoridad, en las comunidades analizadas se constató que este problema más bien se establece en relación con las figuras de autoridad que no son pares de la JCL. En las actividades de trabajo grupal entre jóvenes en clases escolares y en talleres artísticos y laborales, se observó que tienen reglas bastante establecidas y en todos los grupos analizados existen figuras de autoridad legitimadas entre pares. Un rasgo común que al respecto comentaron las psicológicas se refiere a problemas con la figura de autoridad del padre y la madre. Estas profesionales ahondaron en que la JCL proviene de familias en la que el padre está ausente y la madre tiene dificultades en suplir esta figura o el padre representa una figura agresiva, drogadicta y castigadora. En nuestras conversaciones con las psicólogas se asentó el punto de que en la crianza de estas personas jóvenes la familia a cargo de ellas se halla muy ausente en términos de internalización de normas y valores legítimos/legales. Asimismo, existiría una especie de vaivén entre la permisividad con la persona joven y el castigo agresivo cuando comete alguna falta importante. Sobre esta base, se podría hipotetizar que este fenómeno apunta a que la socialización de la que es parte la JCL se acerca mucho más a un modelo de castigo violento y no de convencimiento y persuasión, por lo que la persona joven no haría suyos los patrones de conducta deseados por la sociedad. 
http://doi.org/10.15359/ree.24-3.20

http://www.una.ac.cr/educare

educare@una.ac.cr

Respecto a la gestión institucional, en línea con el trabajo de González Ibarra y Reyes Barragán (2007), el funcionariado identificó la necesidad no cubierta hasta el momento, de contar con desarrollo profesional continuo que lo profesionalice, que considere las especificidades de la población JCL y que cubra un proceso de inducción. Al respecto, la mayoría de pedagogas y talleristas confesaron que no tenían conocimiento previo de las características de la población con la que trabajarían y no contaron con previa inducción por parte de la institución. Algunas pedagogas comentaron que sí tuvieron una inducción, aunque esta fue solo de tres horas sobre algunos datos generales del trabajo que llevarían a cabo.

A pesar de este contexto adverso, en las dos comunidades los resultados de las entrevistas realizadas con la $\mathrm{JCL}$, con personas funcionarias y la observación de actividades, mostraron una serie de prácticas que impactan positivamente en la población JCL y en el personal de la institución, las cuales se expresan en avances en aspectos emocionales y actitudinales. Es decir, prácticas que repercuten positivamente en el desempeño de jóvenes y personas funcionarias. A continuación se detallan estas buenas prácticas tanto a nivel de instrucción como de gestión institucional.

\section{Buenas prácticas de instrucción}

Principalmente estas actividades se desarrollan en los talleres artísticos y laborales, terapias psicológicas y de aprendizaje.

- Realización de evaluaciones cualitativas, las cuales se dirigen a reconocer avances en disciplina, mejora de convivencia y confianza con las personas funcionarias. Asimismo, en estas evaluaciones se lleva a cabo una retroalimentación continua.

- Especialmente en los talleres artísticos y laborales, los grupos de jóvenes poseen márgenes flexibles para diseñar los fines de las actividades. Por ejemplo, en la actividad de teatro participan en el diseño de la obra que se presenta y en el taller de serigrafía cada joven trabaja en la imagen que libremente decide.

- En la observación de las actividades de terapeutas de aprendizaje, psicólogas y talleristas, se enlaza sistemáticamente el contenido formal que se enseña con el conocimiento previo y cotidiano que traen los grupos de jóvenes y se realizan ejercicios lúdicos. Con esta base, se subraya la utilidad concreta de los productos que se realizan, ya sean obras de teatro o manualidades en los talleres laborales como en tejido. En reiteradas ocasiones estas actividades implican un constante trabajo grupal, lo que promueve la colaboración.

- En algunas de estas actividades finalizan un proceso de enseñanza con la construcción de una manualidad u obra que presentan a las comunidades o padres y madres.

- Especialmente en las terapias psicológicas, se trabaja en los aspectos emocionales de jóvenes. Al respecto, se constató que en algunas terapias psicológicas se integra a padres y madres de familia. 
http://doi.org/10.15359/ree.24-3.20

Estas actividades motivan a la población JCL a involucrarse en las tareas y a lograr los productos que se esperan de esta.

\section{Buenas prácticas de gestión institucional}

- En la comunidad de internamiento se apreció que en ocasiones especiales, como días festivos, se lleva a cabo un trabajo transversal entre las actividades, es decir, en cada actividad se desarrolla un tema que está en conexión con los temas desarrollados en las restantes actividades. Las personas funcionarias y la JCL relataron estos episodios con gran entusiasmo y se perciben como actividades memorables.

- Talleristas, pedagogas, psicólogas y terapeutas de aprendizaje, de ambas comunidades, pueden conocer el detalle de los casos que atienden. Cada persona joven sujeta a la LNSIJPA debe seguir un Plan Individualizado de Ejecución (PIE), que en todas las comunidades se elabora en conjunto con las personas funcionarias, jóvenes y su personal de tutoría. Uno de los sentidos de este plan es que la persona joven identifique actividades que realmente le interesan o que su tutoría estima conveniente que curse. Como parte del PIE se confeccionan expedientes que están compuestos por informes que elabora de forma individual cada persona funcionaria que está en contacto con la joven o el joven. En este informe se da cuenta de las características de la persona joven, los objetivos que ha trazado con ella y los avances o retrocesos en torno a estos. Al revisar estos expedientes constatamos que, efectivamente, son bastante completos y otorgan un panorama amplio de las necesidades que se deben trabajar. Otorgan, asimismo, la posibilidad de que cada persona funcionaria conozca con detalle los perfiles, avances y retrocesos de la JCL en distintas áreas y se tomen decisiones basadas en información sistemática.

- Se detectaron buenas prácticas en el manejo de las recompensas por parte de la dirección. Estas eran diversas e iban del reconocimiento verbal de avances en disciplina y respeto de jóvenes, realización de actividades que interesan a la JCL (como taller de fotografía, salidas a museos, proyección de películas), hasta el manejo de la cantidad de alimentos recibidos.

Tal como propusimos, estas actividades de gestión tienen como pilar un trabajo colaborativo, transversal y descansan en objetivos compartidos.

El conjunto de las buenas prácticas de instrucción y gestión institucional se caracterizan por motivar a la JCL y personas funcionarias. De forma sintética, consideramos que se enmarcan en el siguiente proceso: 
http://doi.org/10.15359/ree.24-3.20

http://www.una.ac.cr/educare

educare@una.ac.cr

- En la dimensión instruccional, las jóvenes y los jóvenes se motivan y cumplen las tareas en la medida en que se cuida la pertinencia de lo que se pretende enseñar así como el enlace con los conocimientos previos que trae la JCL; se permite que participen en la definición de los propósitos de aprendizaje y los adapten a sus necesidades; se subrayan sus emociones; se otorga un apoyo y retroalimentación constante y se integra trabajo lúdico cuando es posible. También se apreció que para las jóvenes y los jóvenes la motivación es más relevante cuando por sí se plantean metas a alcanzar y no las conciben como metas impuestas por otras personas.

- En la dimensión de gestión institucional, las personas funcionarias se motivan cuando se toman en cuenta sus opiniones y las de padres y madres en los objetivos institucionales y cuando se lleva a cabo un trabajo transversal entre las actividades (escolares y otras). Además, se identificó un mejor manejo de las relaciones con la JCL cuando las personas funcionarias conocen el detalle de los casos que atienden a través del Plan Individualizado de Ejecución (PIE); y se identificó un control de la disciplina de la JCL por parte de la dirección en el cual se recurre al uso de recompensas que motivan a los grupos de jóvenes.

Estos elementos remiten a la motivación de la JCL y personas funcionarias, y permiten concebir que es posible generar las condiciones sociales de motivación que potencien el aprendizaje de cada JCL. Debido al rol central que consideramos juega la motivación en las buenas prácticas detectadas, el trabajo de campo se complementó constantemente con una revisión de diversas teorías de motivación. A continuación se profundiza en la perspectiva analítica trabajada que permite entender por qué las prácticas señaladas motivan a jóvenes y personas funcionarias.

\section{Motivación, perspectiva analítica}

En una diversidad de escuelas teóricas, la motivación es considerada como un elemento central para lograr aprendizajes integrales, entre estas escuelas destacan: Self direction (Candy, 1991); Transactional control (Dron, 2007), Social constructivist theory (Mclnerney y van Etten, 2003), Value model of motivation (Brophy, 1999); ARCS model of motivation design (Keller, 2010); Motivational framework for culturally responsive teaching (Wlodkowski y Ginsberg, 2010); Self Determination Theory (Ryan y Deci, 2000).

Estas escuelas de motivación se centran en el ámbito educativo y, con distintos matices, definen motivación como aquel componente que permite mantener el involucramiento del estudiantado. Así, la motivación se ha considerado como el motor del aprendizaje (Paris y Turner 1994, citado en Hartnett, 2016). Aquel que permite mantener la focalización y el involucramiento en las actividades, por lo que mejora la persistencia y el rendimiento (Ryan 
y Deci, 2000, citado en Harnett, 2016). En esta línea, de acuerdo con Schunk, Meece y Pintrich (2014, citados en Harnett, 2016, p. 13), la motivación involucra objetivos que proveen el ímpetu para la acción con significado con una dirección deseada, por lo que es un proceso más que un resultado. Esto tiene implicancias en términos de medición de la motivación. Esto es, porque no puede ser observada directamente debe ser inferida desde acciones como elección de tareas, persistencia, esfuerzo y logro, o desde lo que las personas dicen acerca de sí mismas.

Al observar actitudes y actividades positivas respecto al aprendizaje así como autopercepciones favorables en este proceso, se establece que se debe a que el estudiantado se halla motivado. De este modo, la motivación es un constructo teórico para explicar la iniciativa, dirección, intensidad, persistencia y calidad del comportamiento, especialmente del comportamiento orientado hacia objetivos (Brophy, 2010, citado en Harnett, 2016).

Desde la "Self Determination Theory" (SDT) (Ryan y Deci, 2000), se realiza una sistematización de los hallazgos de diversas escuelas de investigación sobre la motivación, y se plantean elementos que conforman entornos sociales que promueven la motivación, a saber: entornos donde el estudiantado alcanza grados importantes de autonomía, se siente competente y fortalece las relaciones con sus pares (pertenencia) (Stirling, 2013).

En la dimensión de la autonomía se enfatiza el grado de control que el estudiantado tiene del proceso de aprendizaje, como pueden ser objetivos, situaciones o trayectorias. La competencia da cuenta del sentido de autoeficacia, es decir, la certeza que tiene el estudiantado de lograr objetivos esperados. Por ende, se refiere al convencimiento de que sí tiene impactos positivos el decidirse a actuar y a la confianza de la persona en sí misma. Por su parte, la pertenencia (relatedness), llama la atención al sentido de comunidad entre pares y de pares con los restantes actores que conforman una situación de enseñanza/aprendizaje. Es la necesidad de cada persona de sentirse seguramente conectada al entorno social y la necesidad de experimentarse a sí misma como valiosa y capaz de respeto (Connell y Wellborn, 1991, citado en Harnett, 2016).

Proponemos que las buenas prácticas identificadas promueven estas dimensiones de motivación. De forma esquemática, se puede decir:

- Las evaluaciones cualitativas, la retroalimentación constante, el enlace entre contenido formal que se enseña con el conocimiento previo y cotidiano que traen los grupos de jóvenes, y el realce de la utilidad concreta de los productos que se realizan potencia el sentido de competencia y confianza en sí misma de la población JCL. Por ejemplo, el hecho de presentar una obra públicamente y recibir reconocimiento promueve el sentido de competencia. En muchos casos, especialmente en las obras de teatro, sus talleristas hablaron del sentido catártico de estas actividades para la comunidad. 
http://doi.org/10.15359/ree.24-3.20

http://www.una.ac.cr/educare

educare@una.ac.cr

- El que los grupos de jóvenes posean márgenes flexibles para diseñar los fines de las actividades influye positivamente en la autonomía en el proceso educativo.

- Los ejercicios lúdicos, trabajo grupal y colaborativo en obras de teatro y talleres laborales promueven el sentido de pertenencia.

Junto a estos elementos, el conjunto de personas funcionarias implicadas en la relación de enseñanza/aprendizaje destacan la importancia de trabajar en los factores emocionales de estos grupos de jóvenes. En general, destacan aspectos identificados en la bibliografía tales como el papel de las emociones en procesar la información que se enfrenta en la configuración educativa; realizar conexiones significativas con la información; y motivarse en mayor medida en el proceso de aprendizaje (Becker y Luthar, 2002; Mathur y Schoenfeld, 2010).

Otro aspecto que se ha desarrollado en la bibliografía sobre motivación corresponde a la motivación intrínseca y extrínseca. Mientras el primer tipo de motivación se refiere a aquella actividad que se lleva a cabo por considerarse en sí misma satisfactoria o interesante, en el segundo se desarrolla una actividad que es vista como un medio para un fin externo a ella. Si bien ha habido una discusión entre la importancia de los tipos de motivación y se han planteado como opuestas y contradictorias; a partir del trabajo de campo consideramos que lo más pertinente es no descuidar la importancia de ambas. Aunque, para que tengan resultados positivos en el aprendizaje, identificamos que es necesario dar espacio a que la motivación (interna o externa) surja de un margen de autodeterminación importante. Es decir, rescatamos la importancia de la motivación internalizada en contra de aquella impuesta. En consecuencia, proponemos que al contrario de concebir la motivación interna y externa como dimensiones opuestas, ambas se pueden complementar cuando los objetivos son internalizados y considerados como propios o autónomos. ${ }^{3}$

Retomando esta complementariedad entre motivación interna y externa, en las buenas prácticas identificadas es común que se conjugue la motivación interna de realizar una actividad que se considera interesante y entretenida por sí misma con la utilidad externa deseada que esta tiene respecto a otros objetivos, por ejemplo, laborales. Desde nuestra perspectiva, entonces, la motivación interna y externa son positivas cuando los grupos de jóvenes las enmarcan en objetivos que perciben elegidos por ellos y no como impuestos.

Ahora, en relación con la importancia de la motivación de personas funcionarias, consideramos que aplican los mismos principios de motivación desarrollados en los procesos de enseñanza/aprendizaje, a saber: autonomía, competencia y pertenencia. Estos principios pueden formar la base comprehensiva de los hallazgos detectados en la amplia bibliografía

\footnotetext{
${ }^{3}$ En términos de motivación impuesta vs autónoma, se puede identificar no solo una motivación externa impuesta sino una motivación externa internalizada (Deci y Ryan, 2012, citados en Hartnett, 2016). Podemos hipotetizar que la motivación interna y la externa internalizada tendrían raíces similares y entrecruzadas.
} 
sobre gestión institucional eficaz y de liderazgo, a saber: liderazgo directivo y distribuido, construcción de una visión común y objetivos compartidos en torno a esta (Acevedo et al., 2017; Maccini y Calvin Cagnon, 2006). Proponemos que a una gestión eficaz subyacen procesos motivacionales en los cuales las personas funcionarias sienten que aportan desde sus experiencias y conocimientos (autonomía), se empoderan y visualizan resultados positivos (eficacia) y se consideran miembros de una comunidad (pertenencia). También hay que agregar que identificamos que las buenas prácticas de gestión detectadas tienen efecto positivo en los objetivos socioeducativos, cuando en el trabajo colaborativo se proponen, conversan y se llegan a acuerdos sobre temas sustantivos relacionados, precisamente, con los procesos socioeducativos de la población JCL.

Finalmente, el conjunto de elementos señalados está en sintonía con el constructivismo social, en donde se puede considerar el aprendizaje como un proceso de participación en actividades compartidas (Hartnett, 2016), es decir, un proceso social. En consecuencia, proponemos que la promoción y potenciación del aprendizaje de la JCL y las personas funcionarias se deben concebir en el marco de contextos sociales que estimulan la motivación y no como procesos aislados o individualistas.

\section{Conclusiones y áreas de mejora}

Enfrentar las problemáticas socioeducativas de la población JCL en internamiento y externamiento, en aras de la reintegración social, requiere contar con datos de las experiencias a las que es expuesta esta población, y diseñar actividades socioeducativas basadas en información robusta y enfocada en ella.

Si bien en los últimos años se ha levantado información sobre la JCL, no se ha profundizado en los perfiles educativos de estos grupos de jóvenes, y en las prácticas de instrucción y gestión institucional se llevan a cabo en las comunidades. Asimismo, no se tiene información sobre los resultados en pruebas estandarizadas, así como en los avances en aspectos emocionales y actitudinales que se están logrando en las comunidades. En consecuencia, se carece de información pública sobre buenas prácticas con resultados positivos en los objetivos socioeducativos planteados en la LNSIJP. Ante esto, la presente investigación se considera como un aporte en la necesidad prioritaria de avanzar en la investigación de buenas prácticas instruccionales y de gestión que pueden ser replicables y adecuarse a contextos particulares, así como identificar prácticas erróneas que es oportuno eliminar.

Ahora bien, a partir del estudio presentado, podemos decir que las comunidades analizadas tratan con jóvenes con dificultades de todo tipo. Son jóvenes que no solo provienen de orígenes sociales desfavorecidos y violentos, sino que poseen problemas de aprendizaje y una considerable falta de motivación respecto a la educación que se les imparte en las escuelas. No 
http://doi.org/10.15359/ree.24-3.20

http://www.una.ac.cr/educare

educare@una.ac.cr

obstante estas dificultades, se identificaron actividades que están logrando resultados positivos en la dimensión socioeducativa de la JCL y en el involucramiento de personas funcionarias. Lo común de estas prácticas es que tienen como núcleo promover la motivación de la JCL y de personas funcionarias. Con esta base, sostenemos que es posible generar las condiciones sociales de motivación que potencien el aprendizaje en cualquier JCL.

Considerando la motivación tanto a escala de la instrucción como de la gestión institucional, se puede decir que promover la motivación implica avanzar en el establecimiento de una comunidad de aprendizaje, es decir, una comunidad en donde las personas aprenden unas de otras. Precisamente, la motivación ha sido identificada como el factor clave en desarrollar y mantener un sentido de comunidad (Hartnett, 2016).

Si bien no se desarrolla en este artículo, es importante considerar que promover la motivación en los distintos actores de una comunidad, y con ello una comunidad de aprendizaje, implica la presencia de relaciones de poder y negociación entre estudiantes, padres y madres, y personas funcionarias. No solo están presentes elementos pedagógicos y didácticos, sino que se manifiestan procesos sociales y políticos que explican alianzas y conflictos.

A continuación se establecen las áreas de mejora que se pueden trabajar en aras de lograr una mayor motivación en la JCL, especialmente en el ámbito de la escuela; y promover la motivación de personas funcionarias.

En la dimensión de instrucción, un problema de motivación relevante se produce en el ámbito de la escuela. Como se señaló, las clases son bastante similares a las que recibe cualquier joven que asiste a una escuela tradicional, es decir, donde la pedagoga desempeña el rol central. Al respecto, algunas pedagogas relataron que en diversas ocasiones que ellas intentaban desarrollar metodologías innovadoras, el estudiantado se divertía y reía, por lo cual las autoridades o los guardias de seguridad controlaban que esto se acabara. En estos recintos está muy presente la idea que la clase debe desarrollarse en un ambiente disciplinado y silencioso. Por lo que corresponde a un ámbito más de control que de enseñanza/aprendizaje.

En consecuencia, es importante evitar el diseño tradicional de clases en la escuela así como su excesivo énfasis formalista. Es necesario que la JCL pueda participar en los contenidos aportando con sus conocimientos previos (autonomía), no ser evaluada solo de forma estandarizada, recibir bastante retroalimentación y realizar productos concretos (competencia), así como participar en trabajo colaborativo (pertenencia). Asimismo, es vital una capacitación docente que permita el manejo de las emociones de esta población. Una forma de potenciar estos aspectos es diseñar clases en donde se realicen distintos momentos de enseñanza formal y participación lúdica o manual y se agreguen momentos donde se presenten productos concretos entre pares, a la comunidad o a padres y madres. 
http://doi.org/10.15359/ree.24-3.20

También se constató una subutilización de la tecnología computacional. Los computadores solo se utilizan para capacitar a la JCL en herramientas básicas como procesadores de textos y no se utilizan enciclopedias multimedia u otros software educativos (que tienen la ventaja de ser interactivos y basarse en una lógica de recompensas constantes). En el caso de la comunidad de internamiento esto también acontece en relación con los televisores que se encuentran en los dormitorios de jóvenes (uno por dormitorio).

Respecto a la gestión institucional, en ambas comunidades existen pocas instancias en las que sus profesionales se reúnen para conversar temas socioeducativos respecto a los casos tratados. La excepción se genera en los días festivos. Es importante sistematizar la transversalidad de contenidos, el trabajo colaborativo y objetivos compartidos de días festivos y llevarlos a la vida cotidiana de las comunidades. También es necesario promover, en mayor medida, el liderazgo distribuido.

Si bien los PIE representan una buena práctica, la elaboración de expedientes demanda bastante tiempo por parte de personas funcionarias en un trabajo que consideran administrativo, además de realizarse en malas condiciones técnicas (hay pocos computadores y desactualizados). Respecto a esto último, se identificó que cada juez interpreta los rubros que conforman los expedientes de distinto modo, por lo cual es común que este solicite que se rehagan, lo cual implica incluso más trabajo.

De especial relevancia es la buena disposición de personas funcionarias en relación con que este carácter de trabajo administrativo e individual se transforme en un trabajo más colectivo, en el que los expedientes se elaboren en conjunto y den pie para conversar aspectos fundamentalmente socioeducativos. En este sentido, estas personas hicieron hincapié en la necesidad de tener mayor contacto y reuniones de trabajo socioeducativo entre sí.

Por otra parte, en ambas comunidades las personas funcionarias identificaron la necesidad no cubierta hasta el momento, de contar con desarrollo profesional continuo que los profesionalice, en un proceso que considere las especificidades de la población JCL y que abarque la inducción. Es prioritario realizar procesos de formación profesional que especialicen a las personas funcionarias en el tratamiento a la JCL.

Asimismo, se identificó la necesidad de generar mayores redes de colaboración con otras agencias de gobierno y organizaciones sociales (salud, rehabilitación, apoyo a los hogares, laborales, etc.). Llamó la atención que diversos apoyos de este tipo, una vez iniciados, no logran continuidad. También se constató una debilidad en el apoyo que se les otorga a la JCL una vez que esta población termina su medida principalmente respecto a diversos servicios laborales, educación técnica, cuidado infantil, consejo familiar, transporte público, etc., o lo que también se conoce como servicios de aftercare (Clark et al., 1993). Por una parte, la ley prohíbe seguir a la joven o al joven una vez que cumple su medida y se evidenció la carencia gubernamental de 
http://doi.org/10.15359/ree.24-3.20

http://www.una.ac.cr/educare

educare@una.ac.cr

este apoyo. Existe un tipo de apoyo a la JCL que es bastante menor y depende de organizaciones civiles que excluyen los casos más problemáticos que han cometido los delitos más graves.

Es pertinente señalar que ambas comunidades analizadas son altamente rígidas en cuanto a las actividades y horarios, lo cual es antagónico a los hallazgos sobre el éxito institucional de experiencias con la JCL.

Por otra parte, se constató que en ambas comunidades se otorgan una serie de talleres de capacitación que se enfocan en aspectos laborales que no requieren elevada formación y que no se cuenta con sistemas de continuidad. Por ejemplo, a los jóvenes de la comunidad de internamiento se los capacita en oficios como serigrafía; empero, los jóvenes difícilmente pueden contar con los materiales en sus domicilios y no existen empresas de este rubro que contemplen la integración de jóvenes de estos grupos. Asimismo, en el taller de teatro, aprenden habilidades sobre las cuales no existen espacios institucionales pensados que les den continuidad como una carrera profesional. Si se tiene en cuenta que además estas comunidades no otorgan certificado laboral alguno que acredite capacitaciones laborales, los grupos egresan de estas comunidades sin oportunidades laborales concretas y relativamente bien pagadas.

Por último, es menester señalar la prioridad de diseñar e implementar un modelo socioeducativo para la JCL, el cual podría recuperar las buenas prácticas de instrucción y de gestión institucional señaladas. En relación con las comunidades analizadas, así como en otros espacios, sería pertinente trabajar en las áreas de mejora identificadas. Especialmente es prioritario integrar a la escuela con las restantes actividades y configurar una comunidad de aprendizaje. Si bien este planteamiento puede concebirse como complejo o poco realista, hay que considerar que se trata de sistematizar y extender las buenas prácticas que ya se están llevando a cabo en las comunidades y desarrollar actividades que demandan las mismas personas funcionarias.

\section{Declaración de Material complementario}

Este artículo tiene disponible como material complementario:

- La versión preprint del artículo en https://doi.org/10.5281/zenodo.3360084

\section{Referencias}

Acevedo, C., Valenti, G. y Aguiñaga, E. (2017). Gestión institucional, involucramiento docente y de padres de familia en escuelas públicas de México. Revista Calidad en la Educación, 46, 53-95. https://doi.org/10.4067/S0718-45652017000100053 
Atkins, T., Bullis, M. y Todis, B. (2005). Converging and diverging service delivery systems in alternative education programs for disabled and non-disabled youth involved in the juvenile justice system. Journal of Correctional Education, 56(3), 253-285.

Azaola, E. (2015). Diagnóstico de las y los adolescentes que cometen delitos graves en México. UNICEF. https://www.casede.org/BibliotecaCasede/Diagnostico adolescentes.pdf

Becker, B. E., y Luthar, S. S. (2002). Social-emotional factors affeting achievement outcomes among disadvantaged students: Closing the achievement gap. Educational Psychologist, 37(4), 197-214. https://doi.org/10.1207/S15326985EP3704 1

Brophy, J. (1999). Toward a model of the value aspects of motivation in education: developing appreciation for particular learning domains and activities. Educational Psychologist, 34(2), 75-85. https://doi.org/10.1207/s15326985ep3402 1

Bullis, M., Yovanoff, P., Mueller, G. y Havel, E. (2002). Life on the "Outs" -Examination of the facilityto-community transition of incarcerated youth. Exceptional Children, 69(1), 7-22. https:// doi.org/10.1177/001440290206900101

Cámara de Diputados del H. Congreso de la Unión. (16 de junio, 2016). Ley Nacional del Sistema Integral de Justicia Penal para Adolescentes. Nueva Ley DOF, pp. 1-82. http://www.diputados. gob.mx/LeyesBiblio/pdf/LNSIJPA.pdf

Candy, P. C. (1991). Self-Direction for lifelong learning. Adult Education Quarterly, 42(3), 192-202. https://doi.org/10.1177/074171369204200307

Centro de Investigaciones y Estudios Superiores en Antropología Social. (2016). Informe especial. Adolescentes: Vulnerabilidad y violencia. CNDH México. http://informe.cndh.org. $\underline{\mathrm{mx} / \text { uploads/menu/30101/Informe adolescentes.pdf }}$

Clark, H. B., Unger, K. V. y Stewart, E. S. (1993). Transition of youth and young adults with emotional/behavioral disorders into employment, education and independent living. Community Alternatives: International Journal of Family Care, 5(2), 19-46.

Dron, J. (2007). Control and constraint in e-learning. Choosing when to choose. Information Science Publishing. https://doi.org/10.4018/978-1-59904-390-6

Elmore, R. (2010). Mejorando la escuela desde la sala de clases. Área de Educción Fundación de Chile. https://fch.cl/wp-content/uploads/2012/08/Libro Elmore.pdf

Foley, R. M. (2001). Academic characteristics of incarcerated youth and correctional educational program. Journal of Emotional and Behavioral Disorders, 9(4), 248-259. https://doi. org/10.1177/106342660100900405 
http://doi.org/10.15359/ree.24-3.20

http://www.una.ac.cr/educare

educare@una.ac.cr

Fullan, M. (2010). The role of the district in tri level reform. En International Encyclopedia of Education (pp. 295-302). https://doi.org/10.1016/B978-0-08-044894-7.01094-0

González Ibarra, J. de D. y Reyes Barragán, L. A. (2007). La administración de justicia de menores en México. La reforma del artículo 18 de la Constitución política de los Estados Unidos Mexicanos. Boletín Mexicano de Derecho Comparado, 40(118), 65-96. http://www.scielo. org.mx/scielo.php?script=sci arttext\&pid=S0041-86332007000100003

Hartnett, M. (2016). Motivation in online education. Springer. https://doi.org/10.1007/978-98110-0700-2

Houchins, D., Puckett-Patterson, D., Crosby, S., Shippen, M. \& Jolivette, K. (2009). Barriers and facilitators to providing incarcerated youth with a quality education. Preventing School Failure, 53(3), 159-166. https://doi.org/10.3200/PSFL.53.3.159-166

Instituto Nacional de Estadística y Geografía. (2018). Resultados de la Primera encuesta nacional de adolescentes en el Sistema de Justicia Penal (ENASJUP) 2017. Comunicado de prensa Núm. 398/18. https://www.inegi.org.mx/contenidos/saladeprensa/boletines/2018/EstSegPub/ ENASJUP2017.pdf

Instituto Nacional de Estadística y Geografía. (2019). El INEGI da a conocer los resultados de la Encuesta nacional de ingresos y gastos de los hogares (ENIGH) 2018. Comunicado de prensa Núm. 384/19. https://www.inegi.org.mx/contenidos/saladeprensa/boletines/2019/ EstSociodemo/enigh2019 07.pdf

Keller, J. M. (2010). Motivational design for learning and performance. The ARCS model approach. Springer. https://doi.org/10.1007/978-1-4419-1250-3

Maccini, P. y Calvin Cagnon, J. (2006). Mathematics instructional practices and assessment accommodations by secondary special and general educators. Exceptional children, 72(2), 217-234. https://doi.org/10.1177/001440290607200206

Magee Quinn, M., Rutherford, R. B., Leone, P. E., Osher, D. M. y Poirier, J. M. (2005). Youth with disabilities in juvenile corrections: A national survey. Exceptional Children, 71(3), 339-345. https://doi.org/10.1177/001440290507100308

Mathur, S. R. y Schoenfeld, N. (2010) Effective instructional practices in juvenile justice facilities. Behavioral Disorders, 36(1), 20-27. https://doi.org/10.1177/019874291003600103

McInerney, D. M. y van Etten, S. (2003). Sociocultural influences and teacher education programs. Information Age Publishing.

Nelson, C. M. (2000). Educating students with emotional and behavioral disorders in the 21st century: Looking through windows, opening doors. Education and treatment of children, 23(3), 204-222. 
http://doi.org/10.15359/ree.24-3.20

http://www.una.ac.cr/educare educare@una.ac.cr

Platt, J., Kaczynski, D. y LeFebvre, R. (1996). Project advance: A comprehensive model program for juvenile incarcerates. Journal of Correctional Education, 47(4), 168-172. https://www. ncjrs.gov/App/AbstractDB/AbstractDBDetails.aspx?id=165036

REINSERTA. (2018). Estudio de factores de riesgo y victimización en adolescentes que cometieron delitos de alto impacto en México. Autor. https://drive.google.com/file/d/1L2UK4HWTGMED49CmovvagXKvUzbnPWG/view

Ryan, R. M. y Deci, E. L. (2000). Self-determination theory and the facilitation of intrinsic motivation, social development, and well-being. American Psychologist, 55(1), 68-78. https://doi.org/10.1037/0003-066X.55.1.68

Stirling, D. (2013). Motivation in education. Aichi Universities English Education Research Journal, 29, 51-72. http://www.learndev.org/dl/Stirling MotEdu.pdf

Wang, X., Blomberg, T. G. y Li, S. D. (2005). Comparison of the educational deficiencies of delinquent and nondelinquent students. Evaluation Review, 29(4), 291-312. https://doi. org/10.1177/0193841X05275389

Wlodkowski, R. J. y Ginsberg, M. B. (2010). Teaching intensive and accelerated courses: Instruction that motivates learning. Jossey-Bass. 